Translokation $\mathrm{t}(4 ; 14)$ oder $\mathrm{t}(14 ; 16)$ definiert wurde. Ohne diese Hochrisiko-ZG betrug das mediane OS 50,5 Monate, mit diesen nur 24,5 Monate [Fonseca R et al. Leukemia. 2009; 23(12):2210-21]. Zudem sind Risikomodelle mit einer Kombination aus Zytogenetik mit ISS oder auch definierten patientenspezifischen Risikofaktoren, wie dem Karnofsky-Performancestatus (KPS), Nierenund Lungenfunktion (Myelom-Komorbiditätsindex [MCI]) mit ISS bekannt, die MMPatienten mit günstigem oder ungüstigem Verlauf unterteilen können. Die aktuelle Arbeit der IMWG ist eine noch umfassendere Analyse einer zuvor von der französischen Intergroupe Francophone du Myelome (IFM)-Studiengruppe vorgeschlagenen Risikostratifizierung, die in 4 Risikogruppen ebenfalls das ISS, ZG und LDH für Myelompatienten geprüft hatte [Moreau $\mathrm{P}$ et al. J Clin Oncol. 2014; 32(20):2173-80]. In der jetzigen IMWG-Kohorte waren alle Risikoparameter (ISS, ZG und LDH) bei $68 \%$ der Gesamtkohorte verfügbar: eine HochrisikoZG lag bei $24 \%$ und eine über der Norm erhöhte LDH bei $13 \%$ der Patienten vor. Mit dem ISS allein betrug die 5-Jahres-OS-Rate bei ISS I, II und III -Patienten 77, 62 und 47\%; auch die Zytogenetik trennte die Patientengruppe, ebenso wie die LDH. Die Kombina- tion aller 3 Risikofaktoren ISS, ZG und LDH erlaubte jedoch eine noch klarere Gruppentrennung mit 5-Jahres-OS-Raten in R-ISS I, II und III von 82,62 und $40 \%$.

Somit stehen die einfachen und überall verfügbaren Risikofaktoren ISS, ZG und LDH zur weiteren Prüfung an: zur Validierung in anderen Zentren, mit Nicht-Studienpatienten und bei different zu diesen in 11 Studienprotokollen behandelten Patienten. Zudem wird ein zukünftiges Ziel sein, dass auch detailliertere genetische Untersuchungen integriert werden, wobei Genexpressionsprofile oder "Single Nucleotide Polymorphismen" (SNP) als komplexere molekulare Analysen noch relativ aufwändig und teuer sind; sie stellen somit noch keine Routine-Risikoparameter dar.

Diskussionspunkte dieser IMWG-Arbeit sind, dass mittels R-ISS nur Studienpatienten innerhalb klinischer Protokolle mit sehr unterschiedlichen Myelomtherapien untersucht wurden. Zudem wurde keine Validierungsanalyse durchgeführt bzw. wurden die Patienten nicht in eine Test- und Validierungsgruppe unterteilt. Außerdem waren die meisten (65\%) der Patienten relativ jung und relevante Chromosomenaberrationen, wie solche von Chromosom 1 oder andere, wurden nicht untersucht. Darüber hinaus war kein Standard-cut-off-Level der LDH bzw. ZG definiert und andere Patientenspezifische Faktoren, wie KPS, Alter, Komorbiditäten - mit relativ kurzem Follow-up der Analyse - wurden nicht berücksichtigt. Somit werden bestätigende Analysen, insbesondere als Validierungsanalyse und bei älteren sowie Nicht-Studienpatienten, mit Spannung zu erwarten sein und der R-ISS gegenüber anderen Risikofaktoren weiter evaluiert werden müssen. Dass die Kombination verschiedener robuster Myelomrisikoparameter aber den Therapeuten unterstützt, aus einem "differenten Mix" prognostisch unterschiedlich verlaufender Myelomerkrankungen eine bestmögliche Subgruppenunterscheidung zu erhalten, wird in dieser IMWG-Arbeit sehr gut veranschaulicht.

Unterstützt von der Deutschen Krebshilfe: DKH $\# 109569+111424$

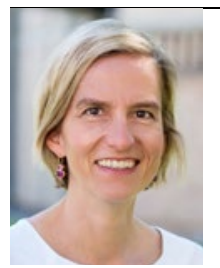

\title{
Verträglichere Erstlinientherapie für Ältere mit multiplem Myelom?
}

\author{
Kommt eine hochdosierte Melphalan-basierte Therapie bei Patienten mit \\ multiplem Myelom (MM) nicht infrage, ist die Kombination MPT-T ein mögli- \\ cher Standard in der Erstlinie. Lenalidomid statt Thalidomid könnte ähnlich \\ wirksam, aber besser verträglich sein.
}

$D_{\mathrm{P}}^{\text {ie }}$ ie Kombination aus Melphalan, Prednison und Lenalidomid (MPRR) wurde in einer Phase-III-Studie mit Nichtunterlegenheitsdesign bei MM-Patienten mit der Kombination Melphalan, Prednison und Thalidomid (MPT-T) verglichen. Im Median wurden 306 zuvor unbehandelte Patienten (median 75,7 Jahre alt) 12,1 Monate behandelt. Bei den 46,6\% von ihnen, die eine Erhaltungstherapie bekommen hatten, betrug der Zeitraum 23,1 Monate.

Das mediane progressionsfreie Überleben (PFS) lag bei 21 Monaten in der MPT-T- und 18,7 Monaten in der MPR-
R-Gruppe (Hazard Ratio 0,84, 95 \%-Konfidenzintervall 0,64-1,09; $\mathrm{p}=0,186)$. Das Gesamtüberleben (OS) betrug 52,6 (MPT-T) im Vergleich zu 47,7 Monaten (MPR-R; p = 0,476). Die Per-ProtocolAnalyse zeigte Ansprechraten von 63,6 und $59,9 \%(\mathrm{p}=0,557)$.

Nebenwirkungen des Grads $\geq 3$ waren in der MPT-T-Gruppe häufiger als in der MPR-R-Gruppe (73,0 vs. 58,0\%; $\mathrm{p}=0,007)$, die Abbruchraten der Therapie wegen Nebenwirkungen betrugen 44,3 und $39,3 \%$. Seltener waren im MPR-R-Arm besonders nichthämatologische Toxizitäten des Grads $\geq 3(40,0$ vs. 59,5\%; $\mathrm{p}=0,001)$. Zweitmalignome traten bei 18 Patienten der MPT-TGruppe und 14 Patienten der mPR-RGruppe auf. Eine Analyse der Lebensqualität fiel zugunsten von MPR-R aus, im FACT-Score für Neurotoxizität zeigte sich eine signifikante Differenz zugunsten von MPR-R ( $p=0,007)$.

Fazit: Eine Behandlung mit MPT-T oder MPR-R bei älteren, zuvor unbehandelten MM-Patienten führte zu vergleichbaren Ergebnissen für das OS, PFS und die Ansprechraten. Es zeigten sich eine bessere neurotoxizitätsbezogene Lebensqualität und eine geringere nichthämatologische Toxizität unter MPR-R. Friederike Klein

Stewart AK et al. Melphalan, prednisone, and thalidomide vs melphalan, prednisone, and lenalidomide (ECOG E1A06) in untreated multiple myeloma. Blood. 2015;126(11):1294-301. 